J. Austral. Math. Soc. (Series A) 44 (1988), 214-224

\title{
VARIATION OF FIXED-POINT AND COINCIDENCE SETS
}

\author{
DAVID GAULD
}

(Received 17 November 1986)

Communicated by J. H. Rubinstein

\begin{abstract}
Topologise the set of continuous self-mappings of a Hausdorff space by the graph topology. When the set of closed subsets of the space is given the upper semi-finite topology then the function which assigns to a map its fixed-point set is continuous. In many familiar cases this is the largest such topology. Related results also hold for the function which assigns to each pair of maps their coincidence set.

1980 Mathematics subject classification (Amer. Math. Soc.): primary 54 H 25; secondary 54 C 35, $54 \mathrm{C} 60$.

Keywords and phrases: fixed-point set, coincidence set, graph topology, upper semi-finite topology, property $W$.
\end{abstract}

\section{Introduction and preliminaries}

Let $X$ and $Y$ be topological spaces and $h: X \rightarrow Y$ a fixed map. If $f: X \rightarrow Y$ is another map then the coincidence set

$$
\psi(f)=\{x \in X \mid f(x)=h(x)\}
$$

is closed in $X$ if $Y$ is Hausdorff. The basic question considered in this paper asks how $\psi_{h}(f)$ changes when $f$ changes. An aspect of this question was considered by Browder [1] and Connett [3] where $f$ is a member of a parametrised family and bounds on the cohomological dimension of the coincidence set are found under certain circumstances.

This paper was presented, in an earlier version, at the 4th International Conference on Topology and its Applications in Dubrovnik, Yugoslavia, on 1st October 1985.

(C) 1988 Australian Mathematical Society 0263-6115/88 \$A2.00+0.00 
Let $\mathcal{F}(X, Y)$ denote the collection of all continuous functions $X \rightarrow Y, \mathcal{F}(X)=$ $\mathcal{F}(X, X)$ and $C(X)$ the collection of all closed subsets of $X$. Continuity of $\psi_{h}: \mathcal{F}(X, Y) \rightarrow C(X)$, for $Y$ Hausdorff, with respect to particular topologies on $\mathcal{F}(X, Y)$ and $C(X)$ will be studied here. The coincidence function

$$
\chi: \mathcal{F}(X, Y) \times \mathcal{F}(X, Y) \rightarrow \mathcal{C}(X),
$$

where

$$
\chi(f, g)=\{x \in X \mid f(x)=g(x)\},
$$

and the fixed-point function $\varphi: \mathcal{F}(X) \rightarrow \mathcal{C}(X)$ with $\varphi=\psi_{h}$, where $h$ is the identity, will also be considered.

Denote by $I$ the closed unit interval $[0,1]$. If $H: X \times I \rightarrow Y$ is continuous, define $\tau_{H}: \mathcal{F}(X, I) \rightarrow \mathcal{F}(X, Y)$ by

$$
\tau_{H}(\alpha)(x)=H(x, \alpha(x)), \quad \forall x \in X,
$$

and set $\mathcal{F}_{H}(X, Y)=\tau_{H}(\mathcal{F}(X, I))$. Define $\psi_{H}: \mathcal{F}_{H}(X, Y) \rightarrow \mathcal{C}(X)$ by

$$
\psi_{H}(f)=\{x \in X \mid f(x)=H(x, 0)\} .
$$

The function $\sigma: \mathcal{F}(X, I) \rightarrow C(X)$ is defined by $\sigma(\alpha)=\alpha^{-1}(0)$.

For any topological space $Z$ and any subset $S$ of the power set of $Z$ the collection

$\{\langle U\rangle \mid U$ is an open subset of $Z\}$,

where $\langle U\rangle=\{S \in S \mid S \subset U\}$ forms a basis for a topology $\tau$ on $S$. Two particular cases are of interest in this paper.

A natural topology on $\mathcal{F}(X, Y)$ is the graph topology, which is the topology $\tau$ above with $Z=X \times Y$ and $S=\mathcal{F}(X, Y)$. This topology is studied in [4] where it is shown that it agrees with the source majorant topology if $X$ is countably compact and normal and $Y$ is metrisable and hence with the compact-open topology if also $X$ is compact.

When $Z=X$ and $S=C(X)$ the topology $\tau$ is called the upper semi-finite topology, introduced by Michael in [7] and studied in [8].

The upper semi-finite topology is so weak that it is not even $T_{1}$, so it is not surprising that $\varphi$ and $\psi_{h}$ are continuous when $\mathcal{F}(X, Y)$ is topologised by the graph topology and $C(X)$ by the upper semi-finite topology. More interesting is the fact that in many familiar circumstances the upper semi-finite topology is the largest topology on $C(X)$ which, when combined with the graph topology on $\mathcal{F}(X, Y)$ or $\mathcal{F}(X)$, is compatible with the continuity of $\psi_{h}$ or $\varphi$. These situations will be explored in Sections 2 and 3.

1.1 Proposition. Suppose $X$ and $Y$ are topological spaces and $f: X \rightarrow Y$ is continuous. Let $Z$ be a set and suppose $g: X \rightarrow Z$ and $h: Y \rightarrow Z$ are functions 
such that $g=h f$. If $\tau$ is the largest topology on $Z$ for which $g$ is continuous then $T$ contains every topology on $Z$ with respect to which $h$ is continuous.

Proof. Any topology on $Z$ for which $h$ is continuous makes $g=h f$ continuous so is contained in $\tau$.

A perfectly normal space is a normal space $X$ in which each closed set is the inverse image of 0 under some continuous function $X \rightarrow I$; equivalently if for each pair $A, B$ of disjoint closed subsets of $X$ there is a continuous function $\alpha: X \rightarrow I$ with $\alpha^{-1}(0)=A$ and $\alpha^{-1}(1)=B$.

1.2 LEMMA. Suppose $X$ is perfectly normal and $U \subset X \times I$ is an open set containing $X \times\{0\}$. Then there is a continuous function $\pi: X \rightarrow(0,1]$ satisfying

$$
\forall x \in X, \quad \forall t \in[0, \pi(x)], \quad(x, t) \in U .
$$

ProOF. Let

$$
V_{n}=\left\{x \in X \mid\{x\} \times\left[0, \frac{1}{n}\right] \subset U\right\} .
$$

Then $\left\{V_{n} \mid n=1,2, \ldots\right\}$ forms an open cover of $X$. By perfect normality this cover shrinks to a closed cover $\left\{W_{n} \mid n=1,2, \ldots\right\}$ of $X$. For each $n$ let

$$
\pi_{n}: X \rightarrow\left[0,1 / 2^{n}\right]
$$

be a continuous function whose support lies in $V_{n}$ and which is $1 / 2^{n}$ on all of $W_{n}$. Define the continuous function $\pi: X \rightarrow(0,1]$ by

$$
\pi(x)=\sum_{n=1}^{\infty} \pi_{n}(x)
$$

It is clear that $\pi$ is positive and if $x \in X$, let $m$ be chosen so that $\pi_{m}(x)>0$ but for all $n<m, \pi_{n}(x)=0$. Then $x \in V_{m}$ and $\pi(x) \leq 1 / 2^{m-1} \leq 1 / m$ so the condition is also satisfied.

1.3 LemMa. Suppose $X$ is perfectly normal, $C \subset X$ is closed, $\alpha_{C}: X \rightarrow I$ is continuous with $\alpha_{C}^{-1}(0)=C$ and $U \subset X \times I$ is an open set containing the graph of $\alpha_{C}$. Then there is an open subset $V$ of $X$ containing $C$ such that for each closed set $D \subset V$ there is continuous function $\alpha_{D}: X \rightarrow I$ whose graph also lies in $U$ with $\alpha_{D}^{-1}(0)=D$.

Proof. Let $W$ be an open subset of $X$ containing $C$ such that $\mathrm{cl} W$ lies in the open set $\{x \in X \mid(x, 0) \in U\}$.

Since $X$ is perfectly normal so is cl $W$ so using Lemma 1.2 to obtain $\pi$ on cl $W$ and normality to extend over $X$, there is a continuous function $\pi: X \rightarrow I$ which is positive on $\operatorname{cl} W$ and satisfies

$$
\{x\} \times[0, \pi(x)] \subset U \quad \forall x \in \operatorname{cl} W .
$$


Let $V=W \cap\left\{x \in X \mid \pi(x)>\alpha_{C}(x)\right\}$. Then $V$ is open and if $x \in C$ then $\alpha_{C}(x)=0$ whereas $\pi(x)>0$ so $x \in V$.

Suppose $D \subset V$ is closed. Then there is a continuous function $\beta: X \rightarrow I$ with $\beta^{-1}(0)=D$ and $\beta(X-V)=1$. Let $\gamma: X \rightarrow I$ be a continuous function with $\gamma\left|X-V=\alpha_{C}\right| X-V$ and $\gamma(C)=1$. Define the continuous function $\alpha_{D}: X \rightarrow I$ by

$$
\alpha_{D}(x)=\min \left\{\max \left\{\pi(x), \alpha_{C}(x)\right\}, \beta(x)\left(\alpha_{C}(x)+\gamma(x)\right) / 2\right\} .
$$

If $x \in V$ then $\pi(x)>\alpha_{C}(x)$ so $\alpha_{D}(x) \leq \pi(x)$ and hence $\left(x, \alpha_{D}(x)\right) \in U$. If instead $x \in X-V$, then $\beta(x)=1$ and $\gamma(x)=\alpha_{C}(x)$ so $\alpha_{D}(x)=\alpha_{C}(x)$ and hence $\left(x, \alpha_{D}(x)\right) \in U$. Thus the graph of $\alpha_{D}$ lies in $U$.

Finally, $\alpha_{D}(x)=0$ if and only if $\pi(x)=\alpha_{C}(x)=0$ or $\beta(x)\left(\alpha_{C}(x)+\gamma(x)\right)=0$. However, when $\alpha_{C}(x)=0, \pi(x)>0$ and $\gamma(x)=1$, so $\alpha_{D}(x)=0$ if and only if $\beta(x)=0$, that is $x \in D$. Thus $\alpha_{D}^{-1}(0)=D$.

1.4 Proposition. Suppose $X$ is perfectly normal. Give $\mathcal{F}(X, I)$ the graph topology. Then the upper semi-finite topology is the largest topology on $C(X)$ with respect to which $\sigma: \mathcal{F}(X, I) \rightarrow C(X)$ is continuous.

PROOF. Firstly suppose that $C(X)$ has the upper semi-finite topology. Then $\sigma$ is continuous, for suppose $\alpha \in \mathcal{F}(X, I)$ and $V \subset X$ is an open set containing $\sigma(\alpha)$. Let

$$
U=(V \times I) \cup[(X-\sigma(\alpha)) \times(0,1]] .
$$

The set $U$ is open in $X \times I$ and it is readily checked that $U$ contains the graph of $\alpha$, that is $\alpha \in\langle U\rangle$. On the other hand, if $\beta \in\langle U\rangle$ then $\sigma(\beta) \subset V$ so $\sigma(\langle U\rangle) \subset V$. Hence $\sigma$ is continuous.

Secondly, suppose $U \subset C(X)$ is such that $\sigma^{-1}(U)$ is open in $\mathcal{F}(X, I)$. To complete the proof it suffices to show that $U$ is upper semi-finite open. Let $C \in U$ and choose a continuous function $\alpha_{C}: X \rightarrow I$ such that $\alpha_{C}^{-1}(0)=C$. Then $\alpha_{C} \in \sigma^{-1}(U)$ so there exists an open set $U \subset X \times I$ such that $\alpha_{C} \in\langle U\rangle \subset \sigma^{-1}(U)$. Let $V$ be the open subset of $X$ given by applying Lemma 1.3. Then $\langle V\rangle$ is an upper semi-finite neighbourhood of $C$. Moreover, if $D \in\langle V\rangle$ then letting $\alpha_{D}: X \rightarrow I$ be as in Lemma 1.3, it follows that $\alpha_{D} \in\langle U\rangle$ and hence that $D=\sigma\left(\alpha_{D}\right) \in U$. Thus $U$ contains $\langle V\rangle$ so $U$ is an upper semi-finite neighbourhood of $C$.

\section{Variation of coincidence gets}

Throughout this section, $X$ is an arbitrary topological space and $Y$ is a Hausdorff space. 
2.1 THEOREM. Let $h: X \rightarrow Y$ be continuous. If $\mathcal{F}(X, Y)$ is topologised by the graph topology and $C(X)$ by the upper semi-finite topology then

$$
\psi_{h}: \mathcal{F}(X, Y) \rightarrow C(X)
$$

is continuous.

Proof. Let $V \subset X$ be open. Set

$$
U=\{(x, y) \in X \times Y \mid x \in V \text { or } y \neq h(x)\} .
$$

It is easily verified that $U$ is open in $X \times Y$ and that $\langle U\rangle=\psi_{h}^{-1}(\langle V\rangle)$.

Theorem 2.1 shows that $\chi$ is continuous in each of the variables separately. While it is unknown whether $\chi$ is continuous in general, under certain circumstances it is continuous.

2.2 THEOREM. Suppose that the topology on $Y$ contains a subtopology $T$ such that either $\tau$ is Hausdorff and the product $X \times Y$ topologised using $T$ on $Y$ is normal or $\tau$ is metrisable. If $\mathcal{F}(X, Y)$ is topologised by the graph topology and $C(X)$ by the upper semi-finite topology then $\chi: \mathcal{F}(X, Y) \times \mathcal{F}(X, Y) \rightarrow C(X)$ is continuous.

Proof. Let $V \subset X$ be open and suppose $f, g \in \mathcal{F}(X, Y)$ are such that $\chi(f, g) \in\langle V\rangle$. To establish the continuity of $\chi$ it suffices to find open sets $U_{1}, U_{2} \subset X \times Y$ containing the graphs of $f, g$ respectively and such that $U_{1} \cap U_{2} \subset V \times Y$.

Since $T$ is Hausdorff it follows that the graphs of $f \mid X-V$ and $g \mid X-V$ are disjoint closed subsets of $X \times Y$ topologised using $\tau$. If the latter space is normal then there are disjoint open subsets $U_{1}^{\prime}$ and $U_{2}^{\prime}$ of $X \times Y$ containing the respective graphs: these sets will also be open when the original topology on $Y$ is used. Let $U_{i}=U_{i}^{\prime} \cup(V \times Y)$.

If $\tau$ is metrisable, let $d$ be a compatible metric on $Y$. Set

$$
\begin{aligned}
& U_{1}=\{(x, y) \in X \times Y \mid x \in V \text { or } d(y, f(x))<d(y, g(x))\}, \\
& U_{2}=\{(x, y) \in X \times Y \mid x \in V \text { or } d(y, f(x))>d(y, g(x))\} .
\end{aligned}
$$

Call a homotopy $H: X \times I \rightarrow Y$ active if

$$
\forall x \in X, \forall t \in(0,1], \quad H(x, t) \neq H(x, 0) .
$$

2.3 Proposition. Suppose $H: X \times I \rightarrow Y$ is continuous. If $\mathcal{F}(X, I)$ and $\mathcal{F}_{H}(X, Y)$ are each given the graph topology then $\tau_{H}: \mathcal{F}(X, I) \rightarrow \mathcal{F}_{H}(X, Y)$ is continuous.

Proof. Let $W \subset X \times Y$ be open and suppose $\alpha \in \tau_{H}^{-1}(\langle W\rangle)$. Since $W$ contains the graph of $\tau_{H}(\alpha)$ then for all $x \in X$, there exist open sets $U_{x} \subset X$ 
and $V_{x} \subset Y$ such that

$$
\left(x, \tau_{H}(\alpha)(x)\right) \in U_{x} \times V_{x} \subset W .
$$

Set

$$
T=\bigcup_{x \in X}\left[\left(U_{x} \times I\right) \cap H^{-1}\left(V_{x}\right)\right] .
$$

The set $T$ is open in $X \times I$. It is claimed that $\alpha \in\langle T\rangle \subset \tau_{H}^{-1}(\langle W\rangle)$.

Suppose $x \in X$. Then $H(x, \alpha(x))=\tau_{H}(\alpha)(x) \in V_{x}$, so

$$
(x, \alpha(x)) \in\left(U_{x} \times I\right) \cap H^{-1}\left(V_{x}\right) \subset T .
$$

Thus $T$ contains the graph of $\alpha$.

Suppose $\beta \in\langle T\rangle$ and $x \in X$. Then $(x, \beta(x)) \in T$ so there exists $\xi \in X$ such that $x \in U_{\xi}$ and $(x, \beta(x)) \in H^{-1}\left(V_{\xi}\right)$. Thus

$$
\left(x, \tau_{H}(\beta)(x)\right) \in U_{\xi} \times V_{\xi} \subset W,
$$

so $\tau_{H}(\beta) \in\langle W\rangle$.

This shows that $\tau_{H}^{-1}(\langle W\rangle)$ is a neighbourhood of $\alpha$ and hence that $\tau_{H}$ is continuous.

2.4 COROLlaRY. Suppose that $X$ is perfectly normal, that $H: X \times I \rightarrow Y$ is active and that $\mathcal{F}_{H}(X, Y)$ is topologised by the graph topology. Then the upper semi-finite topology is the largest topology on $C(X)$ for which

$$
\psi_{H}: \mathcal{F}_{H}(X, Y) \rightarrow C(X)
$$

is continuous.

Proof. Defining $h: X \rightarrow Y$ by $h(x)=H(x, 0)$, it follows that $\psi_{H}$ is the restriction of $\psi_{h}: \mathcal{F}(X, Y) \rightarrow C(X)$ to $\mathcal{F}_{H}(X, Y)$. Thus by Theorem 2.1, $\psi_{H}$ is continuous when $C(X)$ has the upper semi-finite topology.

On the other hand, suppose $\mathcal{F}(X, I)$ also has the graph topology. By Proposition 2.3, $\tau_{H}$ is continuous. Since $H$ is active it follows that $\sigma=\psi_{H} \tau_{H}$. By Proposition 1.4, the upper semi-finite topology is the largest topology on $C(X)$ with respect to which $\sigma$ is continuous. Thus by Proposition 1.1 the upper semifinite topology is the laregest topology on $C(X)$ with respect to which $\psi_{H}$ is continuous.

2.5 COROLLARY. Suppose that $X$ is perfectly normal, that $H: X \times I \rightarrow Y$ is active and that $\mathcal{F}(X, Y)$ is topologised by the graph topology. Define $h: X \rightarrow Y$ by $h(x)=H(x, 0)$. Then the upper semi-finite topology is the largest topology on $C(X)$ for which $\psi_{h}: \mathcal{F}(X, Y) \rightarrow C(X)$ is continuous.

The topological space $X$ has property $W$ (strong) [6] is there is an active deformation $H: X \times I \rightarrow X$. 
The space $\mathbf{R}^{n}$ has property $W$ (strong). Theorem 4.3 of [2] implies that a compact connected topological manifold with Euler character 0 also has this property. It is clear that if $X$ has property $W$ (strong) then so has $X \times Z$ for any space $Z$. The same holds for $E$ is $p: E \rightarrow X$ is a fibre bundle and $X$ is paracompact, locally compact, connected and Hausdorff.

2.6 COROLLARY. Suppose that $X$ is perfectly normal, that $Y$ has property $W$ (strong) and that $\mathcal{F}(X, Y)$ is topologised by the graph topology. Let $h: X \rightarrow Y$ be continuous. Then the upper semi-finite topology is the largest topology on $\mathcal{C}(X)$ for which $\psi_{h}: \mathcal{F}(X, Y) \rightarrow \mathcal{C}(X)$ is continuous.

Proof. Suppose $G: Y \times I \rightarrow Y$ is an active deformation. Define

$$
H: X \times I \rightarrow Y
$$

by $H(x, t)=G(h(x), t)$. Then $H$ is active so the conclusion now follows from Corollary 2.5.

2.7 COROLLARY. Suppose that $X$ is perfectly normal, that $h: X \rightarrow Y$ is a constant function, that $\pi:[0,1] \rightarrow Y$ is a path with $\pi(0)=h(X)$ and $\pi(t) \neq \pi(0)$ for all $t>0$ and that $\mathcal{F}(X, Y)$ is topologised by the graph topology. Then the upper semi-finite topology is the largest topology on $C(X)$ for which

$$
\psi_{h}: \mathcal{F}(X, Y) \rightarrow C(X)
$$

is continuous.

Proof. Define $H: X \times I \rightarrow Y$ by $H(x, t)=\pi(t)$. Then $H$ is active and the conclusion follows from Corollary 2.5 .

2.8 COROLLARY. Suppose that $X$ is perfectly normal, that $Y$ contains a non-constant path and that $\mathcal{F}(X, Y)$ is topologised by the graph topology. Then any topology on $C(X)$ for which $\chi: \mathcal{F}(X, Y) \times \mathcal{F}(X, Y) \rightarrow C(X)$ is continuous is contained in the upper semi-finite topology.

ProOF. Since $Y$ is Hausdorff and contains a non-constant path, there are a point $p \in Y$ and a path $\pi:[0,1] \rightarrow Y$ satisfying $\pi(t)=p$ if and only if $t=0$. Take $h: X \rightarrow Y$ to be the constant map $h(X)=p$. Define

$$
\omega: \mathcal{F}(X, Y) \rightarrow \mathcal{F}(X, Y) \times \mathcal{F}(X, Y)
$$

by $\omega(f)=(f, h)$. Then $\omega$ is continuous and $\psi_{h}=\chi \omega$. Thus any topology on $\mathcal{C}(X)$ for which $\chi$ is continuous also makes $\psi_{h}$ continuous so is contained in the upper semi-finite topology, by Proposition 1.1 and Corollary 2.7. 


\section{Variation of fixed-point sets}

Throughout this section $X$ is a Hausdorff space. Letting $h$ be the identity map on $X$ in Theorem 2.1 and Corollary 2.6 leads immediately to the following corollaries.

3.1 COROLLARY. If $\mathcal{F}(X)$ is topologised by the graph topology and $C(X)$ by the upper semi-finite topology then $\varphi: \mathcal{F}(X) \rightarrow C(X)$ is continuous.

3.2 COROLlaRY. Suppose $X$ is perfectly normal and has property $W$ (strong). Topologise $\mathcal{F}(X)$ by the graph topology. Then the upper semi-finite topology is the largest topology on $C(X)$ for which $\varphi: \mathcal{F}(X) \rightarrow C(X)$ is continuous.

One of the most familiar situations involving fixed points is that where the space under consideration is $B^{n}$, in which case the fixed-point set is non-empty so the conclusion of Corollary 3.2 is false. Let $C^{*}(X)=C(X)-\{\varnothing\}$, and set $\mathcal{F}^{*}(X)=\varphi^{-1} C^{*}(X)$. The graph topology on $\mathcal{F}^{*}(X)$ and the upper semifinite topology on $C^{*}(X)$ will be understood to be the respective restrictions and $\varphi: \mathcal{F}^{*}(X) \rightarrow C^{*}(X)$ will also denote the restriction. By Corollary 3.1, this $\varphi$ is also continuous. Of course $\mathcal{F}^{*}\left(B^{n}\right)=\mathcal{F}\left(B^{n}\right)$ and by [9, Theorem 1] $\varphi \mathcal{F}^{*}\left(B^{n}\right)=C^{*}\left(B^{n}\right)$. It will now be shown that the upper semi-finite topology is the largest topology on $C^{*}\left(B^{n}\right)$ for which $\varphi$ is continuous.

DEFINITION. The topological space $X$ has property $W$ locally uniformly if for each $p \in X$ there is a neighbourhood $N$ of $p$ in $X$ and a continuous function $H: X \times I \times N \rightarrow X$ such that for all $x \in X$, for all $y \in N$, for all $t \in(0,1]$, $H(x, 0, y)=x$ and, if $x \neq y, H(x, t, y) \neq x$. Any space having property $W$ (strong) has property $W$ locally uniformly and any space having property $W$ locally uniformly has property $W[6]$. The spaces $B^{n}$ and $S^{n}$ (all $n$ ) have property $W$ locally uniformly. If $X$ and $Y$ both have property $W$ locally uniformly so has $X \times Y$.

3.3 THEOREM. Suppose $X$ is compact, perfectly normal and has property $W$ locally uniformly. Topologise $\mathcal{F}^{*}(X)$ by the graph topology. Then the upper semifinite topology is the largest topology on $C^{*}(X)$ for which $\varphi: \mathcal{F}^{*}(X) \rightarrow C^{*}(X)$ is continuous.

Proof. Let $U \subset C^{*}(X)$ be such that $\varphi^{-1}(U)$ is open in the graph topology. It must be shown that

for all $C \in U$, there exists open $V \subset X$ with $C \subset V$ such that

$D \in U$ whenever $D$ is a non-empty closed subset of $V$. 
Let $C \in U$. It is claimed that

for all $p \in C$, there exists open $U_{p}, V_{p} \subset X$ with $p \in U_{p}, C \subset V_{p}$, and $D \in U$ for each closed $D \subset V_{p}$ for which $D \cap U_{p} \neq \varnothing$.

Assuming condition $(*)$ for the moment, let $\left\{U_{i} \mid i=1, \ldots, n\right\}$ be a finite subcover of the open cover $\left\{U_{p} \mid p \in C\right\}$ of $C$ and index the corresponding sets $V_{p}$ similarly. Let

$$
V=\left(\bigcup_{i=1}^{n} U_{i}\right) \cap\left(\bigcap_{i=1}^{n} V_{i}\right)
$$

The set $V$ is open and contains $C$. If $D \subset V$ is non-empty and closed then for some $i, D \cap U_{i} \neq \varnothing$. Since also $D \subset V_{i}$, it follows from (*) that $D \in U$ as required.

It remains to verify (*). Let $p \in C$. Since $p$ is fixed for the rest of the proof, the subscript $p$ will be omitted from $U_{p}$ and $V_{p}$. Let $H: X \times I \times N \rightarrow X$ be as in the definition of property $W$ locally uniformly. Let $\alpha_{C}: X \rightarrow I$ be a continuous function with $\alpha_{C}^{-1}(0)=C$ and define $h: X \rightarrow X$ by $h(x)=H\left(x, \alpha_{C}(x), p\right)$. Then $\varphi(h)=C \in U$ so there is an open subset $W$ of $X \times X$ containing the graph of $h$ such that $\langle W\rangle \subset \varphi^{-1}(U)$. Since $X \times X$ is compact and Hausdorff, hence normal, there is an open set $W^{\prime} \subset X \times X$ containing the graph of $h$ such that $\mathrm{cl} W^{\prime} \subset W$.

By compactness of $X \times I$, there is a neighbourhood $U$ of $p$ such that for all $(x, t, y) \in X \times I \times U$, if $(x, H(x, t, p)) \in W^{\prime}$ then $(x, H(x, t, y)) \in W$. Define $\beta: X \times I \rightarrow X \times X$ by $\beta(x, t)=(x, H(x, t, p))$. Then $\beta^{-1}\left(W^{\prime}\right)$ is open in $X \times I$ and contains the graph of $\alpha_{C}$. Let $V$ be the open subset of $X$ obtained by applying Lemma 1.3 with the set $U$ in Lemma 1.3 replaced by $\beta^{-1}\left(W^{\prime}\right)$.

Suppose $D$ is a closed subset of $X$ with $D \subset V$ and $D \cap U \neq \varnothing$, say $y \in D \cap U$. Define $g: X \rightarrow X$ by $g(x)=H\left(x, \alpha_{D}(x), y\right)$ where $\alpha_{D}$ is given by Lemma 1.3. Then $g \in \mathcal{F}^{*}(X)$ and $\varphi(g)=D$. Furthermore, since $\beta^{-1}\left(W^{\prime}\right)$ contains the graph of $\alpha_{D}$ it follows that $\forall x \in X,\left(x, H\left(x, \alpha_{D}(x), p\right)\right) \in W^{\prime}$ and hence $W$ contains the graph of $g$, so that $g \in \varphi^{-1}(\mathcal{U})$. Thus $D=\varphi(g) \in \mathcal{U}$.

3.4 COROLLARY. When $\mathcal{F}\left(S^{n}\right)$ has the graph topology, the upper semi-finite topology is the largest topology on $C\left(S^{n}\right)$ for which $\varphi: \mathcal{F}\left(S^{n}\right) \rightarrow C\left(S^{n}\right)$ is continuous.

Proof. Let $U \subset C\left(S^{n}\right)$ be such that $\varphi^{-1}(U)$ is open in the graph topology and let $C \in \mathcal{U}$. If $C=\varnothing$ then it is clear that $C \in\langle\varnothing\rangle \subset \mathcal{U}$ so that $\mathcal{U}$ is an upper semi-finite neighbourhood of $C$. If $C \neq \varnothing$ then by Theorem 3.3 there is an open set $V \in S^{n}$ with $C \subset V$ such that $D \in \mathcal{U}$ whenever $D$ is a non-empty closed subset of $V$. It suffices to show that $\varnothing \in \mathcal{U}$ also in this case for then $C \in\langle V\rangle \subset U$ and again $U$ is an upper semi-finite neighbourhood of $C$. 
Let $x \in V$, so that $\{x\} \in U$. It is elementary to construct a continuous function (even a homeomorphism) $f: S^{n} \rightarrow S^{n}$ having the single fixed-point $x$ such that arbitrarily close to $f$ there are fixed-point free continuous functions. Since $f \in \varphi^{-1}(U)$, there is a fixed-point free continuous function $g \in \varphi^{-1}(\mathcal{U})$. Thus $\varnothing=\varphi(g) \in U$ as required.

Corollary 2.6 and Theorem 3.3 are not the strongest possible, that is, there are spaces $X$ for which the conclusions remain valid even though the hypotheses are not satisfied. This has already been illustrated by Corollary 3.4. On the other hand, the upper semi-finite topology need not be the largest topology on $C(X)$ or on $\varphi \mathcal{F}(X)$ for which $\varphi$ is continuous. For example, if $X$ is any discrete space then the graph topology on $\mathcal{F}(X)$ is also discrete so $\varphi$ is continuous whatever topology is assigned to $C(X)$; in particular if $C(X)$ is given the discrete topology which is strictly larger than the upper semi-finite topology.

Some fixed-point theorems, such as that of Kakutani [5] apply to multifunctions. A multifunction from $X$ to itself is a function $f: X \rightarrow P(X)$, where $P(X)$ is the power set of $X$. Some authors require $f(x) \neq \varnothing$ for each $x$ but that is not necessary here. The graph of the multifunction $f: X \rightarrow P(X)$ is the set

$$
\{(x, y) \in X \times X \mid y \in f(x)\} .
$$

Let $\mathcal{F}_{m}(X)$ denote the set of all multifunctions from $X$ to itself having closed graph. Under the assumption that $X$ is Hausdorff, each $f \in \mathcal{F}(X)$ has a closed graph so there is a natural inclusion $\mathcal{F}(X) \subset \mathcal{F}_{m}(X)$. Further, the function $\varphi: \mathcal{F}(X) \rightarrow \mathcal{C}(X)$ also extends naturally to a function $\varphi: \mathcal{F}_{m}(X) \rightarrow \mathcal{C}(X)$, where

$$
\varphi(f)=\{x \in X / x \in f(x)\} .
$$

A simple adaptation of the proof of Theorem 2.1 yields the following generalisation of Corollary 3.1.

3.5 THEOREM. If $\mathcal{F}_{m}(X)$ is topologised by the graph topology and $C(X)$ by the upper semi-finite topology then $\varphi: \mathcal{F}_{m}(X) \rightarrow C(X)$ is continuous.

Since $\mathcal{F}(X)$ is a subspace of $\mathcal{F}_{m}(X)$ it is an immediate consequence of $3.2,3.3$ and 3.4 that under the conditions of those results, the upper semi-finite topology is the largest topology for which $\varphi: \mathcal{F}_{m}(X) \rightarrow C(X)$ is continuous.

I wish to thank Dr. M. K. Vamanamurthy for useful discussions during the course of this research. I wish also to thank Dr. M. Mršević for providing me with reference [8] together with a quick translation before I found the American Mathematical Society translation, and long before I found [7]. Finally I wish to thank the Universities of Helsinki and Michigan for providing facilities and the University of Auckland for providing leave during the latter stages of this research. 


\section{References}

[1] F. E. Browder, 'On continuity of fixed points under deformations of continuous mappings', Summa Brasil. Mat. 4 (1960), 183-191.

[2] R. F. Brown and E. Fadell, 'Nonsingular path fields on compact topological manifolds', Proc. Amer. Math. Soc. 16 (1965), 1342-1349.

[3] J. E. Connett, 'On the cohomology of fixed-point sets and coincidence-point sets', Indiana Univ. Math. J. 24 (1975), 627-634.

[4] D. B. Gauld, 'The graph topology for function spaces', Indian J. Math. 18 (1976), 125-132.

[5] S. Kakutani, 'A generalization of Brouwer's fixed point theorem', Duke Math. J. 8 (1941), 457-459.

[6] J. R. Martin, L. G. Oversteegen and E. D. Tumchatyn, 'Fixed point sets of products and cones', Pacific J. Math. 101 (1982), 133-139.

[7] E. Michael, 'Topologies on spaces of subsets', Trans. Amer. Math. Soc. 71 (1951), 152-182.

[8] V. I. Ponomarev, 'A new space of closed sets and multivalued continuous mappings of bicompacta', Mat. Sbornik 48 (90) (1959), 191-212, Amer. Math. Soc. Transl. Ser. 2,38 (1964), 95-118.

[9] H. Robbins, 'Some complements to Brouwer's fixed point theorem', Israel J. Math. 5 (1967), 225-226.

Department of Mathematics and Statistics

University of Auckland

Auckland, New Zealand 